\title{
A Study of Measuring the Effectiveness of the Questionnaire among Physical Education Users
}

KiumarsAzizmalayeri $^{1 *}$, Mohammad Mohammadi ${ }^{2}$

${ }^{1 *}$ Department of Physical Education, Faculty of Literature and Humanities

Malayer University, Malayer, Iran

${ }^{2}$ Department of Educational Sciences, Faculty of Literature and Humanities

Malayer University, Malayer, Iran

\begin{abstract}
The current work is based on drawing data from users' responses against a set of questions. which consists of academic population from universities. The student population and faculty members and staff of the University of Malayer city using random cluster sampling method as statistical sample in this study after examine content validity in order to examine the construct validity exploration factor analysis and for examine reliability Cronbach alpha and for data analyzing SPSS software version were used. According to factor analysis the sport participation questionnaire of members of academic staff were distributed in 8 items which consisting of Psychological, family, social, cultura), administrative, facilities and economical factors. The reliability of questionnaire was calculated as 0/904 by using Cronbach 's alpha correlation coefficient.

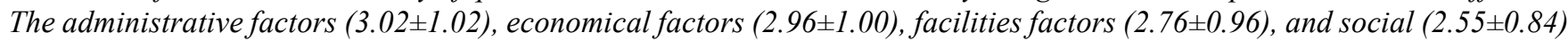
were the most important problems of sports participation of faculty members and university staff. The findings of this work give rise to the responses from a set of questions based on questionnaire which provides a correct yardstick for identification of physical education exercises among the selected target population.
\end{abstract}

Keywords: Standardization, Reliability and Validity, The Obstacles to Sport Participation

Received: 28 April 2019, Revised 11 July 2019, Accepted 20 July 2019

DOI: $10.6025 / \mathrm{pms} / 2019 / 8 / 2 / 52-63$

(C) 2019 DLINE. All Rights Reserved

\section{Introduction}

Living at present world has taken the rapid initiative from human and caused him to movement poverty and that has created for human physical, mental and social problems. Sport as a practical solution and public sport as a means of inexpensive and refreshing can solve this problem effectively (Javadipour \& Saminia, 2013). According to the World Health Organization, increasing physical activity can reduce health care costs and reducing the mortality rate. Exercise programs can be implemented at low costs. It seems to deal with them and encourage people in this regard would be a rational way to improve the general 
health of society. One of the major discussions in planning sport field is the necessity existing programs with the needs and problems of balance of individual and group. Exercise is one of the ways which people can overcome emotional, social and psychological stress of living in today's turbulent world. Studies have shown that people who exercise regularly, less than others are experiencing cardiovascular disorders, suffer less nervous stress and are more confidence, they are also more optimistic in life and are less depressed (Sonnentag S \& et al, 2008). Sports participation considered as an essential component of the planning process in various areas, including educational, cultural and sports activities. Because in today's competitive world, one of the most important organizational resources in universities is human resources and only organizations can survive in this turbulent world that can best use of their resources and ignoring their mental and physical health can have long-term negative consequences of social, economic, political consequently irreparable damage to society so it is necessary for all universities that apply all efforts to encourage faculty and staff to exercise to improve physical health. Therefore holding each program including sports programs requires a precise understanding of existing requirements and needs, because the lack of attention to people's expectations of customers, causing a waste of money, time and manpower (Ahmadi \& Kargar, 2013).the purpose of present study is combining the previous measuring tools of the barriers of sport participation in order to development and manufacture of tools for measuring the barriers to sport participation of faculty and staff, as well as validity and reliability of the questionnaire. Therefore, the role of exercise and physical activity as a strategy which could potentially assist in the goals of health, social and economic, is very important (SeyedAmeri, 2012). Abram et al (2013) reported in their study people who have a higher education level in comparison with those who have lower education level perceive fewer barriers because educated people have more knowledge and understanding about the advantages and benefits of exercise and physical activity, as well as better compatibility with health promotion programs in comparison with other people. From the perspective of researchers one of the inhibitor causes of exercise, is lack of awareness in advantages and benefits of people and insufficient informing to the community (Aghaee et al, 2012). Therefore it seems that examine the levels of activity and the barriers of sport participation of individuals is specific to each country with economic level and the level of special culture (Eligani et al, 2012). In a study recommended a social norms and security as one of the key aspects that may affect the participation exercise and also these results showed that health plans are popular among people more than other projects (Parsamehr et al, 2013).from the view of sociolinguistics, sports culture is a subculture often called sports culture. This culture includes beliefs, values, norms, habits and made the sport is human social (Physical Education Organization, 2005). Najafi (2014) concluded in his research that barriers to sport participation of mental, personal, cultural, social and family are respectively have the most and less priority and administrative, facilities and economical barriers are without impact in sport participation of staffs of Malayer city organizational employees. Therefore, understanding the needs and attitudes of Faculty members and staff of universities in planning matters relating to the development of sports and physical education is essential. High performance and efficiency of faculty members and staff of university, is something that like other resources and human resources of the society requires enjoying them from mental and physical health and peace and joy that providing these requirements is possible only in having healthy and dynamic living and efficient use of time and leisure in order to provide the public health. One of the major problems of the country's universities is lower faculty members and staff participation in sporting activities. It should be noted that university faculty members and staff are the most effective segments of society. Therefore the important issue for doing the current study is that, how it can be possible according to present condition and by desirable organizing and planning took an important step to investigate the factors influencing sport participation and physical activity and regular exercise and consistent development of our universities, faculty members and staff. But so far, few studies have been done in this field and barriers to sport participation Faculty members and staff have not been fully identified. Hence given the importance of measuring the barriers of sport participation and the faculty members and university staff and need to a proper tool for measuring this concept in sport participation forced the faculty members and university staff to do standardized questionnaires of the barriers of sport participation of faculty members and the staff of universities and the present research has done with this purpose.

\section{Methods}

The research method is descriptive and of survey studies. The population of this study comprised of Faculty members and staff. Jackson (2003) believes that in exploratory analysis factor the minimum sample size of 200 is defensible (Jackson, 2003). In this research for analyzing the barriers of sport participation of faculty members and staff of university the number of 379 were selected among 4000 individuals of faculty members and staff of Hamadan university with random cluster as statistical sample. The tool of the research was researcher made questionnaire that its variables its variables were obtained through investigation prestigious university textbooks, scientific papers published in prestigious journals and interviews with and sport management professors and experts. Among 100 obtained variables for measuring the barriers of sport participation 39 variables which had more repetition were identified as items of questionnaire of sport barriers. After extracting these variables, 10 individuals of

Progress in Machines and Systems Volume 8 Number 2 October 2019 53 
experts and professors of sport management confirmed the face and content validity of the questionnaires. The reliability of questionnaire of the barriers of faculty member and staff of university in a guide study were investigated by a 30 member group of faculty members and staff. And its amount was calculated 0/904 by using Cronbach's alpha correlation coefficient and finally the questionnaire in two sections of individual characteristics (age, marital status, profession, level of education) and the main section of questionnaire items (Psychological, personal, family, social, cultural, administrative, facilities and economic) were distributed among research samples. Among 390 distributed questionnaires 370 were returned. Descriptive statistics (Frequency, mean and standard deviation) was used for evaluation of demographic characteristics and determine the amount of variables importance. And exploratory factor analysis with orthogonal rotation were used for examine the aspects and barriers of sport barriers and the reliability of research tools. The Kolmogorov-Smirnov was used to calculate the internal consistency of the subscales. All data were analyzed with SPSS23 version 23 and Expert Choice 11.

\section{Findings}

The results of descriptive results of the study revealed that the average age of 379 respondents was $37 / 84$ years and the number of 69/9 percent were men and 30/1 of them were women which among them 75/2 percent are married and 24/8 are single about employment status results indicated that 200 individuals 55/6 were formally employed, 112 individuals $25 / 4$ percent were contractual employed and 38 individuals 19 percent were contract employment. Also 92 individuals 24/3 percent had $\mathrm{PhD}$ degree, 144 individuals 38 percent had MA degree, 111 individuals 29/3 percent has bachelor degree, 11 people $2 / 9$ percent had associated degree, 21 number 5/5 percent had diploma and 114 individuals 30/1 percent were formal, 39 people 10/3 percent were official-experimental, 59 individuals $15 / 6$ percent were contract and finally 149 percent were contractual and the average service duration was 10/84 year, the organizational position of 18 people $4 / 7$ percent was master, the organizational position 18 people, 4/7 percent was supervisor, organizational position 18 people, $4 / 7$ percent was supervisor, the organizational position of 23 individuals $6 / 1$ percent was assistance, the organizational position of 42 individuals $11 / 1$ percent was management, the organizational position of 18 people $4 / 7$ were master, the organizational position of 15 people $33 / 3$ percent was faculty membership, the organizational position of 19 people $27 / 4$ percent was service and finally 59 people $15 / 6$ percent had the other organizational positions. According to table 1 the amount of Kisermi and Oklin test was meaningful that identifies that there is correlation between items and indicates providing required condition for doing confirmatory factor analysis in this research. To identify the internal structure exploratory factor analysis with orthogonal rotation was used. Before implementing factor analysis the researcher used KMO test for sampling and understating whether the number of items is enough for predicting each component. Also the Bartlet test was used for determine if the items have meaningful relationship for providing a rational basis for factor analysis. According to Leech report, Brett and Morgan (2005) KMO must be more than (\%70) and Brtlet test must be less than (\%5) (Leech et al, 2005). Therefore, having regard to the assumptions of the test, the obtained results were the confirmatory of factor analysis. Table 1 shows the test results of Bartlet and KMO.

\begin{tabular}{|l|c|}
\hline \multicolumn{1}{|c|}{ Variable } & Results \\
\hline KMO test for Proportional of sample size & 0.871 \\
\hline Bartlet test for correctness of factors separation & 0.001 \\
\hline
\end{tabular}

Table 1. The results of factor analysis of Bartlet test and KMO questionnaire

Table 1 indicates that the size of samples is appropriate for analysis $(K M O=0 / 871)$ and the Bartlet test is meaningful statistically $(P \leq 0 / 05)$ which indicates correlation among variables. The results of test indicated that the exploratory factor analysis is proportionate with the items of questionnaire. Then by implementing the command of orthogonal rotation obtained 9 factor for explain the samples behavior. Then correlation within each of the components in the specified factors was calculated through Cronbach's alpha. Despite recommendations of Leech Brett and Morgan (2005) who have recommended Cronbach's alpha coefficient above $0 / 7$ and according to exploratory nature of the study factors that had higher internal correlation from 0/639 were selected as proper factors for explain the barriers of sport participation. Therefore 8 factors of (Psychological, personal, family, social, cultural, administrative, facilities and economic) remained. These 8 factors devoted 58/68 percent of variance to its table the scree plot curve were used for the number of used factors. This curve showed that choosing two factors for variables is sufficient. Because after sixth factor a shift in the slope of charts is observed which indicates the lower of impact and change in the variance by seventh factors to the next but because we in this study we considered 8 subsidiaries and a contributor to the 
questionnaire, here we choose 8 factor as main factors for analyzing exploratory factor.

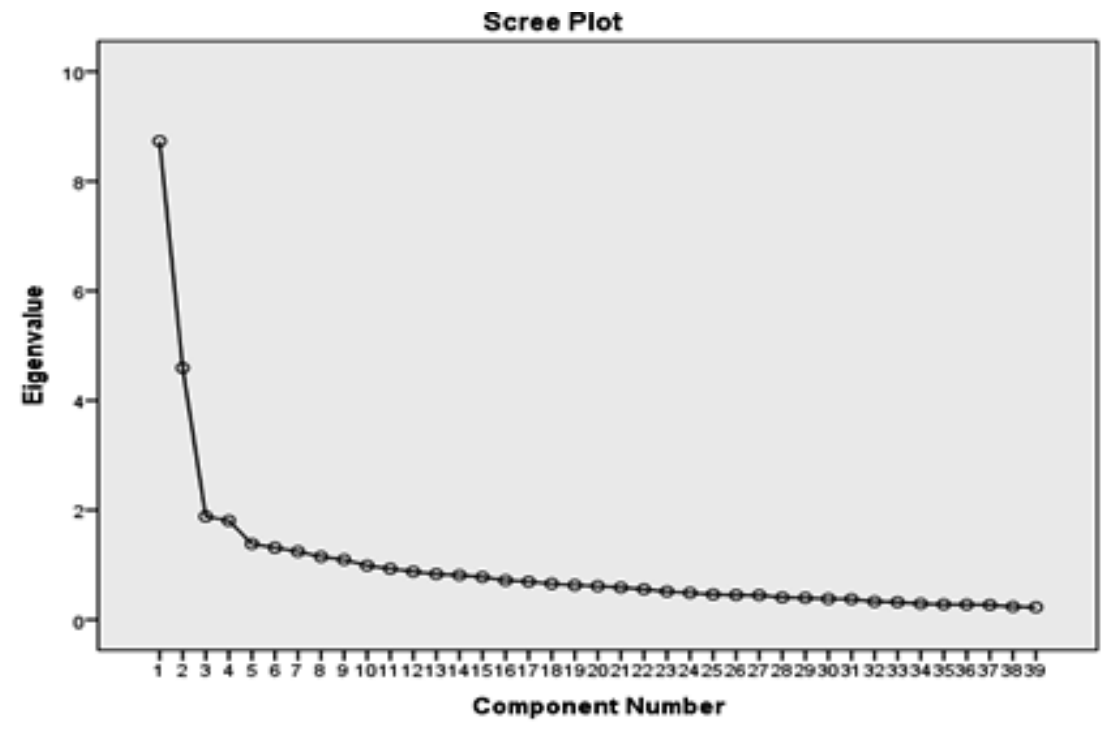

Chart 1. The chart of Scree Plot of factors

In table it can be seen that each factor how much of the total variance included

\begin{tabular}{|l|c|c|c|}
\hline Components & The special volume & The percent of variance & The cumulative percentage \\
\hline First factor & $8 / 730$ & $22 / 384$ & $22 / 384$ \\
\hline Second factor & $4 / 594$ & $11 / 778$ & $34 / 162$ \\
\hline Third factor & $1 / 883$ & $4 / 829$ & $38 / 991$ \\
\hline Fourth factor & $1 / 807$ & $4 / 634$ & $43 / 626$ \\
\hline Fifth factor & $1 / 384$ & $3 / 548$ & $47 / 174$ \\
\hline Sixth factor & $1 / 313$ & $3 / 366$ & $50 / 540$ \\
\hline Seventh factor & $1 / 244$ & $3 / 190$ & $53 / 730$ \\
\hline Eighth factor & $1 / 148$ & $2 / 944$ & $56 / 674$ \\
\hline
\end{tabular}

Table 2. The table of Factor analysis

After the ninth stage of Varimax rotation these coefficients are converted as follows:

We can see that the "special values" and "percentage of total variance described by agents" after the rotation have been modified and closer to each other. An analysis result of variables has come in Table 3. In this table the darker homes reflects belonging that question to a relevant factor as it can be seen in that tale no item belongs to factors of second to eighth which is insufficient distribution. Therefore, we use varimax rotation 9 stage rotation and modulate this situation. The table 5 shows the results of Varimax rotation which is more appropriate. According to varimax rotation the first factor justify 60/12 percent of variance changes which the largest absolute value of the coefficient elements related to the first component column belongs to highlight items and include statements of administrative and social obstacles. The second factor which 8/021 percent of variance changes belong to it is the biggest absolute value of factor analysis associated with it belongs to highlight items of the column of second component and includes the items of family and cultural barriers in the third factor 7/612 percent of variance changes belonged to it the largest absolute value of factor analysis associated with that belongs to highlight items of third 


\begin{tabular}{|l|c|c|c|}
\hline \multicolumn{1}{|c|}{ Components } & $\begin{array}{l}\text { The special } \\
\text { volume }\end{array}$ & $\begin{array}{c}\text { The percent of } \\
\text { variance }\end{array}$ & $\begin{array}{c}\text { The cumulative } \\
\text { percentage }\end{array}$ \\
\hline First factor (Administrative and social obstacles) & $4 / 781$ & $12 / 260$ & $12 / 260$ \\
\hline Second factor (Family and cultural barriers) & $3 / 128$ & $8 / 021$ & $20 / 280$ \\
\hline Third factor (Personal and psychological barriers) & $2 / 969$ & $7 / 612$ & $27 / 892$ \\
\hline Fourth factor (economical barriers) & $2 / 655$ & $6 / 807$ & $34 / 699$ \\
\hline Fifth factor (the personal and psychological barriers) & $2 / 651$ & $6 / 798$ & $41 / 497$ \\
\hline Sixth factor(facilities barriers) & $2 / 584$ & $6 / 626$ & $48 / 122$ \\
\hline Seventh factor (social barriers) & $1 / 865$ & $4 / 783$ & $52 / 905$ \\
\hline Eighth factor (family barriers) & $1 / 470$ & $3 / 769$ & $56 / 674$ \\
\hline
\end{tabular}

Table 3. The table of factor analysis after the ninth stage Varimax rotation

component and includes items of personal and psychological barriers in the fourth factor that 6/807 percent of variance changes is depended to it the largest absolute value of factor analysis belongs to highlight items of fourth column component and includes economic barriers items in the fifth factor which 6/798 of variance changes depended to it, the largest absolute value of analysis factor belongs to highlight items of the column of fifth component and includes personal and psychological items in the sixth factor which 6/626 percent of variance changes depends to it the largest absolute values of related analysis factor belongs to highlight items of seventh component column and includes social barriers items.in the eighth factor that 3/769 percent of variance changes depends to it the largest absolute value related analysis factor belongs to highlighted items of eighth component and includes family barriers items generally these eight factors 56/674 percent of changes includes all data variance which considered as a proper value.

\begin{tabular}{|l|l|l|l|l|l|l|l|l|}
\hline Items & \multicolumn{7}{|c|}{ Factor coefficient } \\
\hline ej2 & First & Second & Third & Fourth & Fifth & Sixth & Seventh & Eighth \\
\hline em2 & 0.613 & 0.004 & 0.059 & 0.004 & 0.021 & 0.092 & -0.101 & -0.246 \\
\hline fa2 & 0.607 & -0.182 & -0.360 & 0.083 & -0.080 & 0.135 & 0.196 & -0.098 \\
\hline em3 & 0.586 & -0.253 & 0.327 & -0.162 & -0.105 & 0.002 & -0.020 & -0.239 \\
\hline em4 & 0.579 & -0.223 & -0.262 & 0.032 & -0.311 & 0.119 & -0.274 & -0.117 \\
\hline fa4 & 0.575 & -0.129 & -0.380 & 0.014 & -0.309 & 0.080 & 0.053 & -0.147 \\
\hline fa1 & 0.552 & 0.000 & 0.164 & -0.108 & -0.214 & 0.003 & 0.112 & -0.390 \\
\hline egh2 & 0.549 & 0.333 & -0.087 & -0.176 & -0.166 & -0.327 & -0.147 & 0.089 \\
\hline ej4 & 0.547 & -0.301 & -0.305 & 0.213 & 0.211 & -0.082 & 0.116 & 0.060 \\
\hline ed2 & 0.543 & -0.337 & 0.293 & -0.018 & -0.017 & -0.005 & -0.007 & 0.047 \\
\hline ej5 & 0.534 & -0.489 & 0.221 & -0.177 & 0.063 & -0.144 & 0.113 & -0.022 \\
\hline em5 & 0.526 & 0.094 & 0.169 & -0.055 & -0.011 & 0.171 & -0.307 & 0.356 \\
\hline ed4 & 0.521 & -0.085 & -0.410 & 0.104 & -0.378 & -0.112 & -0.074 & 0.005 \\
\hline egh3 & 0.512 & -0.458 & 0.132 & -0.214 & -0.026 & -0.078 & -0.021 & -0.111 \\
\hline ed3 & 0.512 & -0.208 & -0.412 & 0.122 & 0.372 & 0.013 & 0.183 & 0.122 \\
\hline
\end{tabular}




\begin{tabular}{|l|l|l|l|l|l|l|l|l|}
\hline fa5 & 0.505 & -0.396 & 0.169 & -0.098 & -0.020 & -0.017 & 0.189 & -0.026 \\
\hline em1 & 0.497 & 0.157 & 0.072 & -0.175 & -0.105 & -0.026 & -0.371 & 0.266 \\
\hline egh1 & 0.494 & -0.236 & -0.144 & 0.265 & -0.108 & 0.010 & -0.242 & 0.137 \\
\hline ej3 & 0.493 & -0.147 & -0.470 & 0.061 & 0.224 & 0.180 & 0.038 & 0.132 \\
\hline ej1 & 0.484 & -0.120 & 0.247 & -0.007 & -0.008 & 0.365 & -0.238 & 0.273 \\
\hline kh4 & 0.479 & 0.253 & 0.169 & -0.041 & 0.014 & -0.123 & 0.327 & 0.367 \\
\hline kh3 & 0.467 & 0.370 & -0.032 & 0.140 & 0.038 & -0.368 & 0.114 & -0.107 \\
\hline kh5 & 0.465 & 0.344 & -0.041 & -0.142 & 0.402 & -0.201 & -0.140 & -0.067 \\
\hline r2 & 0.455 & 0.219 & 0.137 & -0.013 & 0.139 & 0.125 & -0.298 & -0.128 \\
\hline ed1 & 0.396 & 0.553 & 0.103 & 0.133 & -0.116 & -0.072 & 0.070 & -0.184 \\
\hline kh1 & 0.471 & -0.532 & 0.293 & -0.087 & -0.017 & -0.083 & 0.147 & 0.077 \\
\hline ed5 & 0.414 & 0.520 & -0.067 & -0.259 & 0.210 & -0.112 & 0.030 & 0.176 \\
\hline r1 & 0.487 & -0.508 & 0.234 & -0.198 & 0.062 & 0.003 & 0.247 & 0.119 \\
\hline sh2 & 0.295 & 0.505 & 0.010 & -0.057 & -0.116 & 0.230 & 0.359 & 0.118 \\
\hline
\end{tabular}

Table 4. Results of items factor analysis

\begin{tabular}{|l|l|l|l|l|l|l|l|c|}
\hline Items & \multicolumn{7}{|c|}{ Factor coefficient } \\
\hline & First & Second & Third & Fourth & Fifth & Sixth & Seventh & Eighth \\
\hline ed5 & 0.767 & -0.038 & 0.000 & 0.233 & -0.031 & -0.025 & 0.092 & -0.114 \\
\hline ed2 & 0.765 & 0.085 & -0.056 & 0.176 & -0.059 & 0.065 & 0.061 & 0.063 \\
\hline ed1 & 0.761 & -0.035 & -0.130 & 0.147 & 0.037 & 0.038 & 0.109 & -0.021 \\
\hline fa2 & 0.684 & 0.081 & 0.166 & -0.097 & 0.050 & 0.226 & 0.116 & 0.176 \\
\hline ed4 & 0.673 & 0.068 & -0.013 & 0.103 & -0.146 & 0.214 & 0.100 & 0.098 \\
\hline ed3 & 0.661 & -0.003 & 0.033 & 0.175 & 0.044 & 0.124 & 0.032 & -0.014 \\
\hline ej4 & 0.629 & 0.020 & -0.008 & 0.101 & 0.124 & 0.100 & 0.241 & 0.090 \\
\hline fa4 & 0.482 & 0.153 & 0.317 & -0.132 & 0.158 & 0.359 & -0.096 & 0.128 \\
\hline ej2 & 0.352 & 0.141 & 0.315 & 0.097 & 0.123 & 0.301 & 0.146 & 0.283 \\
\hline fa3 & -0.036 & 0.750 & 0.098 & -0.022 & 0.190 & 0.215 & 0.061 & 0.013 \\
\hline fa1 & 0.114 & 0.675 & 0.119 & 0.014 & 0.133 & 0.276 & 0.192 & -0.035 \\
\hline kh1 & -0.029 & 0.597 & 0.407 & 0.158 & 0.092 & -0.115 & 0.163 & -0.068 \\
\hline kh3 & 0.063 & 0.556 & 0.320 & 0.210 & 0.007 & -0.086 & 0.119 & 0.320 \\
\hline kh4 & 0.076 & 0.543 & 0.135 & 0.126 & 0.377 & 0.120 & -0.133 & 0.167 \\
\hline sh5 & 0.024 & -0.377 & -0.196 & 0.174 & 0.374 & -0.001 & 0.169 & 0.369 \\
\hline r5 & 0.036 & 0.026 & 0.659 & 0.027 & 0.128 & 0.189 & 0.152 & 0.036 \\
\hline r3 & -0.058 & 0.068 & 0.641 & 0.046 & 0.149 & 0.027 & -0.045 & -0.104 \\
\hline sh2 & -0.038 & 0.344 & 0.610 & 0.105 & 0.063 & -0.020 & 0.170 & 0.079 \\
\hline
\end{tabular}




\begin{tabular}{|l|l|l|l|l|l|l|l|l|}
\hline sh4 & 0.038 & 0.307 & 0.544 & 0.037 & 0.011 & 0.028 & 0.160 & 0.135 \\
\hline r1 & -0.039 & 0.174 & 0.523 & 0.037 & 0.378 & 0.010 & 0.004 & -0.325 \\
\hline egh3 & 0.198 & 0.099 & 0.110 & 0.773 & 0.008 & 0.113 & 0.004 & 0.074 \\
\hline egh1 & 0.090 & 0.040 & 0.193 & 0.672 & -0.054 & 0.258 & 0.149 & 0.012 \\
\hline egh2 & 0.298 & 0.086 & -0.040 & 0.647 & 0.092 & 0.230 & 0.010 & 0.134 \\
\hline egh4 & 0.435 & -0.016 & -0.140 & 0.503 & -0.023 & 0.140 & 0.124 & 0.128 \\
\hline sh1 & 0.059 & 0.205 & 0.093 & 0.029 & 0.761 & 0.072 & 0.055 & -0.095 \\
\hline $\mathbf{r 4}$ & -0.025 & -0.013 & 0.178 & -0.024 & 0.694 & 0.029 & 0.117 & 0.152 \\
\hline sh3 & -0.027 & 0.181 & 0.118 & 0.022 & 0.655 & 0.079 & 0.145 & 0.174 \\
\hline $\mathbf{r 2}$ & -0.030 & 0.373 & 0.366 & -0.122 & 0.466 & 0.162 & -0.036 & 0.125 \\
\hline
\end{tabular}

Table 5. Results of factor analysis of items after 9 rotation stages

\section{Results of Confirmatory Factor Analysis}

According to Fornel and Larker (1981) recommendations the amount of load factor for the observed variables must be greater than $0 / 5$ the obtained results from confirmatory factor analysis has showed in table 6 . The results of confirmatory factor analysis based on major and minor version has been drawn.

\begin{tabular}{|c|c|c|}
\hline Factors and items & General Factor load & Partial factor load \\
\hline \multicolumn{3}{|l|}{ Psychological barriers } \\
\hline Negative impressions and shyness & $0 / 555$ & $0 / 546$ \\
\hline Fear of being judged by others when attending the community & $0 / 549$ & $0 / 538$ \\
\hline having unpleasant feeling when sweating & $0 / 557$ & $0 / 366$ \\
\hline Lack of motivation to exercise. & $0 / 552$ & $0 / 355$ \\
\hline Having the unfavorable feeling to sports spaces & $0 / 513$ & $0 / 422$ \\
\hline \multicolumn{3}{|l|}{ Personal barriers } \\
\hline Having unpleasant experience and the lack of sufficient skills in sports & $0 / 652$ & $0 / 630$ \\
\hline Lack of knowledge of the benefits of exercise and a fitness program & $0 / 542$ & $0 / 638$ \\
\hline Tense, hard and boring of sports activities & $0 / 534$ & $0 / 734$ \\
\hline Physical weakness & $0 / 538$ & $0 / 663$ \\
\hline To be busy and not having enough time to exercise. & $0 / 516$ & $0 / 555$ \\
\hline \multicolumn{3}{|l|}{ Family barriers } \\
\hline Family negative attitude towards sports activities & $0 / 601$ & $0 / 510$ \\
\hline Existence many obligations and responsibilities of family & $0 / 674$ & $0 / 205$ \\
\hline Family lack of consensus about the proposed hours of sports activities & $0 / 583$ & $0 / 631$ \\
\hline Lack of family agreed to participate in sports trips or competitions & $0 / 537$ & $0 / 487$ \\
\hline Lack of mobility and physical inactivity family members & $0 / 515$ & $0 / 335$ \\
\hline
\end{tabular}




\begin{tabular}{|c|c|c|}
\hline \multicolumn{3}{|l|}{ Social barriers } \\
\hline Negative attitudes of colleagues toward exercise & $0 / 581$ & $0 / 345$ \\
\hline Lack of access to skilled trainers & $0 / 559$ & $0 / 467$ \\
\hline Lack of participation of friends and colleagues in sports activities & $0 / 574$ & $0 / 519$ \\
\hline Discouraging the exercise by the university administration & $0 / 597$ & $0 / 499$ \\
\hline Lack of familiarity with diversity Health Promotion & $0 / 568$ & $0 / 443$ \\
\hline \multicolumn{3}{|l|}{ Cultural barriers } \\
\hline lack of consistent of sports coverage with religious observance & $0 / 615$ & $0 / 746$ \\
\hline Lack of considering the development of sports culture in the workplace & $0 / 610$ & $0 / 770$ \\
\hline Fears of damage to religious beliefs & $0 / 659$ & $0 / 772$ \\
\hline The restriction to wear sports clothes from living place to sport place & $0 / 553$ & $0 / 693$ \\
\hline \multirow{2}{*}{$\begin{array}{l}\text { Failure to inform the public about the benefits of exercise and physical activity by } \\
\text { corporate media }\end{array}$} & $0 / 527$ & $0 / 479$ \\
\hline & $0 / 615$ & $0 / 746$ \\
\hline \multicolumn{3}{|l|}{ Administrative barriers } \\
\hline Weakness of sport planning in the University & $0 / 633$ & $0 / 605$ \\
\hline Lack of instruction or directive that requires universities to provide support for sports & $0 / 642$ & $0 / 702$ \\
\hline Not paying attention to women's sports & $0 / 487$ & $0 / 551$ \\
\hline Lack of attention to filling medical file for annually monitoring health & $0 / 555$ & $0 / 576$ \\
\hline Lack of educational programs related to sport & $0 / 667$ & $0 / 662$ \\
\hline \multicolumn{3}{|l|}{ Facilities barriers } \\
\hline Lack of access to spaces and sports facilities outside of office hours & $0 / 580$ & $0 / 382$ \\
\hline Inappropriate health status of sport stadium and facilities & $0 / 611$ & $0 / 579$ \\
\hline Lack of a variety of and athletic fields sport and facilities & $0 / 654$ & $0 / 614$ \\
\hline Inadequate Equipment and means of sports & $0 / 618$ & $0 / 610$ \\
\hline Inadequate heating and cooling systems in indoor sports facilities & $0 / 619$ & $0 / 576$ \\
\hline \multicolumn{3}{|l|}{ Economic barriers } \\
\hline Expensive exercise equipment & $0 / 590$ & $0 / 561$ \\
\hline Exercise spending in other areas & $0 / 596$ & $0 / 648$ \\
\hline The high entrance fee for sports facilities & $0 / 677$ & $0 / 674$ \\
\hline Lack of financial support from faculty and staff exercise & $0 / 514$ & $0 / 506$ \\
\hline
\end{tabular}

Table 6. Exploratory factor analysis 
According to the results of the factor analysis for total or partial loadings greater than $0 / 5$ can be stated that those items indicating factors and in other words they are capable of measuring considered structure.

\section{Discussion and Conclusion}

One of the important characteristics of each test is its validity and reliability. This study was conducted with the purpose to investigate the validity and reliability barriers to sport participation of faculty members and staff at universities in this study designing items of sport participation barriers has done while investigating academic books and numerous scientific articles and interviews with sports management, experts and faculty and staff attended. This research study showed that barriers to sport participation of 100 variables, 39 were of higher value. Based on the factor analysis the obstacles were classified in the eight psychological, personal, family, cultural, administrative, social and economic. Lack of a validate and reliable tool in order to measuring obstacles of sport participation of faculty members of universities and effective factors on their participation in order to create practical strategies in this regard and access to more productivity and success resulted that present research determines the validity and reliability the questionnaire of obstacles of sport participation of faculty members and university staff that have reliable factors and indexes in order to using for researchers and other managers in this regard Borno (1999) by doing a research stated that ,since the basis of doing any research is using validate and reliable measuring tools and construe and explanation of research results depended on validity of applied tools , thus researchers must be confident from the validity of tools that they are using in their research. The most important and essential stage in determine validity of questionnaires with different cultural contents was determine the validity of the structure of questionnaire and in this field the confirmatory factor analysis is the best tool. Barbara and William (2005) expressed that in confirmatory factor analysis, certain theoretical models compare with each other and, in fact, a useful method for reviewing the proper tools of the research is used. In examining the confirmatory the general and partial factor analysis would supplied. This means that when we measure factor analysis of each component in comparison with all items of questionnaire the general factor load is obtained and when measure loading factor of each component in comparison with items of the same component, the partial loading factor is obtained. When an item in terms of general factor load or in terms of partial factor load have an amount under $0 / 5$, then that items would remove from questionnaire items. In examining the items of psychological barriers we can explain that in terms of general factor load the item of "lack of motivation for exercise" has the highest factor load. And also in terms of partial factor load of the item" Negative impressions and shyness "has the highest partially factor load. Here any of the items will not be removed and they are able in the measuring considered structures. Since the anxiety of cognitive state is related to worry and negative thoughts of people while the physical state of anxiety relates to moment to moment changes in perceived physiological activity. Physical state of anxiety is not essentially change in person's physical activity but is the perceived of person from such change. Therefore in addition to experience negative thoughts and comprehend pomological activities the amount of person's beliefs and the ability to deal with the challenges is also an important component from state anxiety (Dalman \& Livaz, 2010) also Manl et al (2001) stated about full time employees in their study that lack of interest and unaware of programs are not considered as the obstacles of participation in the leisure time activities. But obstacles divided into three categories of interpersonal, intrapersonal and structural which include different sub variables. Kranford et al,1993) presented a hierarchical and showed that inhibiting factors are placed in the a hierarchy of decision making process and a person experience these factors in the hierarchy of individual inhibiting factors, interpersonal and structural, respectively. They are believed that participation is dependent on inhibitory factors rather Lack of inhibiting factors means of participation. Results of this research is consistent with results of Ghodratnama et al (2013), Najafitokhoshke (2014), RahimeMehdizadeh et al (2014), Najaf Aghaee et al (2012), Harvi et al (2007) in reviewing the items of personal barriers can explain that in terms of factor load all items of "Misery Business and not having enough time to exercise "has the highest load factor. Also in terms of partially factor load the item of "hang unpleasant experiences and lack of sufficient skill in the exercise" has the highest partially factor load. Here none of the items removed and they are capable in measuring considered structures. Despite increasing public awareness about the effects of the consequences of immobility, based on the available evidence only a small percentage of the population of civil servants embracing sports programs. With the consultation exercise can be used as a way to eliminate some of the psychological barriers to effective participation in physical activity. So if not increase their level of physical exercise, they may have diminished their quality of life or even sometimes physical problems or mental suffering and reduce their effectiveness (Tontes Mateo, 2005). So try to eliminate some of the negative attitudes toward participation in sports activities is very important. Results of research consistent with results of Syros Ahmadi et al (2013) and Dalman \& Livaiz (2010). In examine the items of family barriers it can be explain that in terms of general factor load it item "existence a lot of commitments and family responsibilities" has the highest factor load. Also in terms of partially factor load the item "negative attitude of family toward sport activities" has the highest partially factor load. Here none of the items removed. And they can be helpful in the considered structure. The most important issue in the family socialization process through interactions with each other and impressionable of family members from each other. Therefore, it is necessary to note 
that in the process of sports socialization the acceptance among family members have a special importance that cause to affect the person (Hashemi $\mathrm{tal}, 2011$ ) according to conducted researches it is one of the initial requirements for introductory familiar with supportive exercise that family applies from the person to be able while introductory entrance to sports world decide depend on interest and ability toward continuing or withdraw a sports field. Studies has shown that continues and long-term education from the media, educational centers ... seems that improve this aspect (Prince et al, 2012) results of this research is consistent with results of Aghaee et al (2012), Mehdizadeh et al (2011) and Dalman and Liwaiz (2010) in study the items of social barriers it can be explain that in terms of factor load the item "Discouraging the exercise by the university administration. "Has the highest factor load also in terms of partially factor load "negative attitudes of colleagues toward sport" has the highest factor load. Here none of the items would remove and they are helpful in measuring considered structure. Despite increasing public awareness about the effects of the consequences of immobility, only a small percentage of the population of civil servants based on the available evidence embracing sports programs. Bagherzadeh et al 2001 reported in their research implementing different sport programs will increase the interest of people to the sport so one of the good strategies is removing some personal barriers can be holding such competitions and sport festivals.so if not increase their physical activities may their quality and quantity of their life would be diminished or even occur some physical and mental problems for them and would reduce their effectiveness (Tontes Mateo, 2005). results of this research is consistent with results of AyubEslami et al (2013), Parsamehr et al (2013), Najafi to khoshke (2014) and VandanDrish et al (2012) to examine the items of cultural obstacles it can be explain that in terms of factor load of item "not considering the development of sports culture in the workplace "has the highest factor load, also in terms of partially factor load the item "fear to damage to religious beliefs" has the highest partially factor load. Here none of the items removed and they can be helpful in measuring considered structure. The complexity of culture subject is due to its interdisciplinary and cross-sectorial as well as human and social factors in it (Ahmadi, 2009) in this regard Adelkhani et al (2012) reported that the cultural capital of persons is effective in the attitude and their tendency to physical activity. The spiritual level or cultural basis includes values, norms, behaviors, beliefs and attitudes. And its material and tangible level related to products such as goods or services and cultural tools such as book, press, art, cinema, informing, promotion etc. (Moeinaddini et al, 2012). Sport as one of the secondary institutions of modern society, come along with functions such as health, leisure, identity, prosperity and... Therefore exercise, like all other phenomena in society is influenced by social and cultural factors, some scientists know sports as a cultural affair to communicate in a common language of the world (Locker, 2002) results of this research is consistent with results of SyyedHabiboallahmirxafori et al (2009) and Najafi to khoshke (2014). To examine the items of administrative obstacles can be explain such that in terms of factor load the item of "The lack of educational programs related to sport. "Has the highest factor load also in terms of partial factor load the item "Lack of instruction or directive that requires universities to provide support for sports" has the highest factor load. Here none of the items removed and they can be helpful in measuring considered structures. Management systems, agencies and organizations is very important to organizational goals or offices, including the management systems authoritative, organized, caring, counseling and human relations (Ramazaninejhad et al, 2011). Given that organizational life associated with stress, work-related stress and fatigue. In such circumstances, experts and behavioral sciences at the office, mention entertainment sport as a means of effective and suitable for recreation, increase vitality and joy. To in addition to increasing capacity for work, be effective to grow and develop ethical aspects, mental and social (Rahime, Mahdizadeh et al, 2014) according to the first model the contingency management that Fred Fidlerproposed in this pattern two main concept of employee orientation and work orientation have been considered. A leader that characterized as an employee-oriented his work focuses on aspects of the work relationship (Ramazaninejhad et al, 2011) results of research consistent with results of Eligani et al (2011), Prince et al (2012), Hang and Hamrfis (2012).to examine the items of facilities obstacles it can be explain that in terms of factor load the item "Lack of access to suitable spaces and sports facilities outside of office hours "has the highest factor load, also in terms of partial factor load the item "The inadequate health status of sport places" has the highest partially factor load. Here none of the items would remove. And they can be helpful in the measuring considered structures it should be noted that existence of sport facilities has the highest role in the attraction and sport participation of citizens in public and leisure sport programs and increasing quality in the offering place services and sport facilities can increase the satisfaction and sport participation of people. The findings of researches of planning team for national Australian institute in 2000, know effective the proximity of sport places to place of citizens residence in increasing sport participations of citizens.in findings of (Salis et al,1998) sport places as the most basic hardware part in the physical Education Organization and sport and a part of the facilities and human organizations which directly and not directly affects its surrounding environment. Sport facilities in addition to its positive consequences such as increased prosperity, employment, communications, transportation, enjoyment of facilities and services, etc., may result in undesirable consequences such as environmental problems, cultural problems, social, and so on (Ali Hosseini et al, 2014) results of research consistent with results of Mehdizadeh et al(2014), Najafi to khoshke (2014). to examine the items of economic obstacles it can be explain that in terms of general factor load the item "The lack of financial support from faculty and staff in sporting activities " has the highest factor load and also in terms of partial factor load the item "The high cost to entrance sports facilities "has the highest partial factor load. Also during

Progress in Machines and Systems Volume 8 Number 2 October 2019 
these years the economic aspects has always been one of the most important state matters. One thing that will help to accomplish this is to examine the factors influencing people's attitudes towards this issue. Among these factors we can mention the kind of person's motivation toward sport participation that indicates their attitude toward public sport and some effective factors on it such as the social-economic status (Eslami et al, 2013) in another research indicated that factors such as financial, facilities, expertise human resource problems and restrictions related to membership in teams are examples of sport participation obstacles. Therefore economic obstacles considered as obstacles that have positive impact on reducing effect of sport of faculty member and staffs of Malayer University. In this aspect obstacles such as low income, high entrance to sport places, investing sport costs in another places, expensive exercise equipment's... are effective on lack participation of faculty members and university staff. The mentioned cases indicates that people who have higher education level and better socio-economic status regularly do exercise which its reason is having more awareness toward physical education (Hang \& Hamfris, 2012) because the most evident effects of economic factor on sport participation is its effect on choosing desirable sport and the higher class of people in terms of economic they would have lower constriction to choose type of sport. And should not be ignored that the maintenance staff is very important in offices (Motmani et al, 2012). Results of this study is consistent with Dalman \& Liwaiz (2010), Eligani et al (2011) and Vandandrish et al (2011) here no items removed. And they can helpful in measuring considered structure. Finally due to obtained results from this research , the questionnaire of the barriers of sport participation of faculty members and university staff is a reliable and validate tool that increases the international support of validity of this model. And we can use this tool to assess the barriers and incentives used their presence in universities and obtain reliable and stable results. In other words, the data collected through this questionnaire, provide credible information about barriers to sport participation. In the final conclusion, it can be stated that the questionnaire was a multidimensional instrument consists of psychological, social, cultural, familial, personal, administrative, and economic barriers with confirming construct validity. Therefore it is applicable in studies efforts on spectators. Given the extensive applicable of this questionnaire in different countries, it is recommended that other researchers in our country in order to measuring the obstacles of sport participation of faculty members and the universities of our country to use this questionnaire to obtain more validate results.

\section{References}

[1] Aghaee, N., Fattahian, Nafisa. (2012). A comparative analysis of the barriers to sport participation of athletes and untrained teachers Crawford pyramid model: (a case study of teachers in Hamedan), Journal of Research in Management and Motor Behavior, 2 (4) 174-161.

[2] Ahmad, Cirus., Kargar, Said. (2013). Attitudes of women's sports participation and social factors - psychological influence on it, Journal of Applied Sociology, 25 (52) 210-189.

[3] Ahmad, Syed Abdul Hamid. (2009). Mnshvrfrhngy sports Islamic Republic of Iran, Tehran: Center for Culture and Arts (SID), cultural management team, in order to mobilize the sport.

[4] Chenge-Ya, Huang (2005). A study on the participative motives of silver- haired Taichichuan participants in.

[5] Crawford, D., Jackson, E., Godbey, G. (1991). A hierachical model of leisure constraints. Leisure Sciences, 13, 309-320.

[6] Dollman., Lewis, R. (2010). The impact of socioeconomic position on sport participation among South Australian youth. Journal of Science and Medicine in Sport, 1, 318-322.

[7] El- Gilany, A. H., Badawi, K., El-Khawaga, G., Awadalla, N. (2011). Physical activity profile of students in mansoura University, Egypt. Eastern Mediterraneam Health Journal. 17 (8) 694-702.

[8] Harvey, J., Levesque, M., Donnelly. P. (2007). Sport volunteerism and social capital. Sociology of Sport Journal, 24 (2) 206223.

[9] Hashemi, Syed Zia., Moradi, R. (2012). Role of the Family in the Socialization of Sports, Journal of Sociology, 11 (4) 168-143.

[10] Huang, H., Brad, R., Humphreys. (2012). Sports participation and happiness: Evidence from US micro data. Journal of Economic Psychology, 33, 776-793.

[11] Ibrahim, S., Karim, A. N., Oon, L. N., Ngah, W. Z. (2013). Perceived physical activity barriers related to body weight status and sociodemographic factors among Malaysian men in Klang Valley. BMC public health. 13. 275.

[12] Islami, ayoub., Mahmoud, Ahmed., Khabiri, Muhammad., NajafianRazavi, S. M. (2013). The role of socio - economic (SES) in motivating citizen participation in sport - recreational, Journal of Applied Research in Sport Management, 2 (3) 104-89. 
[13] Islamic Republic of Iran Physical Education Organization (2005). Comparative studies of the development of sports culture, Tehran, green apple.

[14] Jackson, D.L. (2003). Revisiting sample size and number of parameter estimates: Some support for the N: q

[15] Javadipour, Muhammad, Sami A., M. (2013), sport in Iran and vision, strategy and future plans, Applied research in sport management, (4), Ss30-21.

[16] Kaohsiung city, thesis.

[17] Laker, Anthony. (2002). The Sociology of Sport and physical education, Rutledge.

[18] Leech, N., Barret, K., Morgan, G. (2005). SPSS for intermediate statistics, 2nd Eddition.

[19] Mannel, R. C., Hubbard, J. (2001). Testing Models of the leisure constraint negotiation process in a corporate Employee Recreation setting, Human Resources services \& Department of Recreation and leisure studies, University of waterloo.

[20] Zadeh, Mehdi., Rahima., Andam, R., (2014). Strategies for the development of sport in Iranian universities, Journal of Sport Management, 22, 38-15.

[21] Mir Ghafuri, SeyedHabibollah., Sayaditoranlo, Hussein., Mirfakhredini, Syed Haider. (2009). An analysis of the issues and problems affecting women's participation in sports activities (Case Study: Girls Yazd University), Journal of Sport Management, Numbers 1, 100-82.

[22] MoinaldiniJavad., Hematkhah, A. R. (2012). Social factors - cultural effects tend to sport for all citizens in Kerman case studies, Journal of Urban Studies, . 2 (3) 178-149.

[23] Motameni, AlirezaHemmati., RaminMoradi., Hadi. (2014). Identify priorities obstacles facing women in sports activities, Sports Management Studies, 24, 130-111.

[24] Najafitokhoshke, Giti. (2014). The Pathology of sports participation Malayer city employees, Master Thesis, Islamic Azad University of Boroujerd.

[25] Parsamehr, Mehraban., Blgvryan, Mastaneh., Saeed Madani, M. (2013). Study on relationship between social capital and sports participation among adolescents, Journal of Sport Management, 17, 127-109.

[26] Prins, Richard, G., Sigrid, M., Mohnen., Frank, J., Van lenthe., Johannes, Brug., Anke, Oenema. (2012). Are neighbourhood social capital and availavlity of sports facilities related to sports participation among Dutch adolescents? International Journal of Behavioral Nutrition and Physical Activity, 9 (90) 2-11.

[27] Ramezaninejad, Rahim., Mohebi, Hamid., HEMATINEZHAD, Mehr Ali. (2009). Needs Assessment of physical education in primary schools in the country, Research in Sport Science, Number 22, Spring turn, 38-27.

[28] Salllis, J. F., Buman, A., Pratt, M. (1998). Environmental and Policy Interventions to Promote Physical Activity, American Journal of Preventive Medicine.

[29] Seyyedameri, Mir Hossein., Muhammad algh, GorbanBerdy. (2012). Explain the techniques to attract and increase citizen participation in sport and recreation programs (publication of case studies Urmia), Journal of Management Studies sport and Movement Science, 2(4) 32-23.

[30] Adelkhany, Amin., VaezMousavi, Seyed Mohammad Kazem., Farahani, A. (2012), Cultural Capital of participants in the sport (policies and procedures), Fslnammh Parliament and strategy, Year 19, (69), 32 -5.

[31] Ali Hosseini, B., Bahr al-Hassan., Nia Hosseini, Seyed Reza. (2014). The relationship between motives for sport participation and commitment among veterans and disabled athletes, Journal of Emerging Trends in Sport Management, 2(6), 54-43.

[32] Sonnentag, S., Niessen, C. (2008). Staying vigorous until work is over: The role of trait vigour, day-specific work experiences and recovery. Journal of Occupational and Organizational Psychology, 81(3) 435-58.

[33] Tonts, Matthew. (2005). Competitive sport and social capital in rural Australia. Journal of Rural Studies 21, 137-149.

[34] Vandendriessche, J. B., Barbara, F. R. Vandorpe., Roel, V. (2012). Variation in Sport Participation, Fitness and Motor Coordination with Socioeconomic Status among Flemish Children. Pediatric Exercise Science, 24, 113-12. 